\title{
DISEÑO DEL PULSO ÓPTIMO DE RIEGO EN EL CULTIVO DE FRESA CON EL MODELO NUMÉRICO HYDRUS 2D
}

\author{
García Morillo, J. ${ }^{1}$, Rodríguez-Díaz, J.A. ${ }^{1}$, Martín Arroyo, M. ${ }^{1}$, Camacho, E. ${ }^{1}$, Montesinos \\ Barrios, P. 1
}

1 Universidad de Córdoba. Departamento de Agronomía, Campus de Excelencia Internacional Agroalimentario, ceiA3. E-mails: g62gamoj@uco.es, jarodriguez@uco.es, o02maarm@uco.es, ecamacho@uco.es, pmontesinos@uco.es

\section{Resumen}

El cultivo intensivo de fresa, que se desarrolla en macrotúneles de plástico sobre lomos acolchados, demanda volúmenes importantes de agua para satisfacer las necesidades hídricas del cultivo así como para labores de preparación del suelo o alomado y riego de apoyo en la fase de plantación. El riego de la fresa es especialmente complejo dado que es un cultivo que se produce bajo plástico en suelos muy arenosos (habitualmente con un contenido de arena superior al $90 \%$ ), con escasa capacidad de retención de agua. A pesar de esta dificultad, no es habitual que exista una programación del riego basada en la medida de las principales variables que afectan al cálculo de las necesidades hídricas del cultivo. En este trabajo se proponen medidas de mejora en el manejo del riego, basadas en la determinación del pulso óptimo que evite las pérdidas por percolación en tres fases de desarrollo del cultivo. Para ello se ha utilizado el modelo de simulación del movimiento del agua en el suelo Hydrus 2D. Se han simulado diferentes estrategias de riego para aplicar la $\mathrm{ET}_{\mathrm{c}}$ del cultivo con emisores de $5 \mathrm{I} \cdot \mathrm{h}^{-1} \cdot \mathrm{m}$ en tres etapas diferentes del cultivo, inicio, mediados, y final de campaña y en las que por término medio se necesitan tiempos de riego que oscilan entre los 10-20 minutos al inicio de la campaña y 40-60 minutos al final de campaña.

\section{1- Introducción. Objetivo del trabajo}

El riego por goteo está considerado como uno de los sistemas de riego más eficientes dado que permite tener un mayor control sobre las aplicaciones de agua, fertilizantes y pesticidas. El conocimiento del perímetro de bulbo húmedo que genera es crítico para manejar el sistema de riego de manera eficiente (Samadianfard, et al., 2012).

Los modelos numéricos son una herramienta útil para analizar la evolución del bulbo húmedo durante el riego a fin de determinar estrategias de manejo del riego por goteo que determinen el tiempo de riego y optimicen la eficiencia del uso del agua. HYDRUS 2D (Šimůnek et al, 1999) es un modelo que permite simular el movimiento del agua, temperatura y solutos en dos dimensiones en un suelo no saturado. La precisión del modelo para simular el movimiento del agua en el suelo ha sido puesta de manifiesto en varios estudios, por ejemplo Šimůnek et al. (2008).

El objetivo de este trabajo es aplicar el modelo numérico Hydrus 2D para estudiar la dinámica del agua en el cultivo de fresa bajo diferentes estrategias de riego. Para ello se ha calibrado el modelo con datos medidos de bulbo húmedo en una urna en laboratorio. Posteriormente se han simulado estrategias de riego orientadas a aplicar la $\mathrm{ET}_{\mathrm{c}}$, definiendo el pulso óptimo de riego que evite las pérdidas de agua por percolación. 


\section{Materiales y métodos}

Para determinar la estrategia de riego óptima para las condiciones de producción de la fresa, se ha simulado el movimiento del agua en un lomo tipo para un periodo de una semana de duración en tres etapas del cultivo claramente diferenciadas, inicio (noviembreenero), mediados (febrero-marzo) y finales de campaña (abril-junio).

La ecuación que gobierna el movimiento del flujo de agua en un suelo no saturado en el modelo es la de Richards, incluyendo el término "sumidero" (S) para considerar la absorción del agua por el sistema radicular en función del estrés hídrico y/o salino. Se introduce mediante este término un modelo de respuesta de la planta a la humedad del suelo (Šimůnek et al., 2006). A continuación se muestra esta ecuación para dos dimensiones:

$$
\frac{\partial \theta}{\partial t}=\frac{\partial}{\partial x} \cdot\left[K(h) \frac{\partial h}{\partial x}\right]+\frac{\partial}{\partial z}\left[K(h) \frac{\partial h}{\partial z}+K(h)\right]-S
$$

En la que $\theta$ representa el contenido volumétrico de agua $\left[\mathrm{L}^{3} \mathrm{~L}^{-3}\right]$, $\mathrm{h}$ el potencial de presión [L], t el tiempo [T], x la coordinada horizontal, $z$ la coordinada vertical y $\mathrm{K}$ es la conductividad hidráulica [L. $\mathrm{T}^{-1}$ ]. En Hydrus el término $S$ representa el volumen de agua extraído por las raíces en la unidad de volumen de suelo por unidad de tiempo. Para ello emplea una función compleja propuesta por Feddes et al. (1978).

El modelo resuelve la ecuación de Richards mediante la función paramétrica de van Genuchten $(1980,1987)$ que relaciona la humedad y el potencial del suelo mediante la siguiente ecuación:

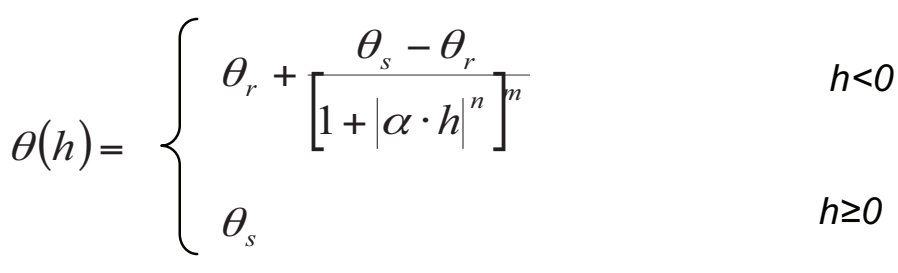

donde $\theta_{\mathrm{s}}$ es la humedad en saturación del suelo, $\theta_{\mathrm{r}}$ es la humedad residual del suelo, $\mathrm{K}_{\mathrm{s}}$ es la conductividad hidráulica saturada del suelo, y $\mathrm{m}, \mathrm{n}$ y a son valores empíricos que afectan a la forma de la curva de retención y que como simplificación se asume que $m=1-$ $(1 / n)$.

La conductividad hidráulica no saturada $\mathrm{K}(\mathrm{h})$ se determina mediante la siguiente expresión (Mualem, 1976):

$$
K(h)=K_{s} \cdot S_{e}^{L} \cdot\left[1-\left(1-S_{e}^{1 / m}\right)^{m}\right]^{2}
$$

donde el término $S_{e}$ es el contenido de agua adimensional o también llamado contenido de agua efectivo, $K_{s}$ es la conductividad hidráulica saturada del suelo y $L$ es un parámetro empírico relacionado con la conectividad entre los poros.

El modelo Hydrus-2D resuelve la ecuación de Richards (ecuaciones 1 a 3) sujeta a determinadas condiciones de contorno aplicando el método de los elementos finitos bajo un esquema de Galerkin. El procedimiento de cálculo se explica en detalle en Šimůnek et al. (1999). 
Los términos $\theta_{r}, \theta_{s}, \alpha, n, y L$ que aparecen en las ecuaciones expuestas son los llamados parámetros hidráulicos del suelo, valores que determinan la forma de la curva de retención y la conductividad hidráulica, bases de la ecuación del movimiento del flujo en suelo no saturado de Richards. Estos parámetros tienen un significado físico aunque en muchas ocasiones se utilizan meramente como parámetros de ajuste (Arbat Pujolràs, 2005).

$\theta_{\mathrm{r}, \text { y }} \theta_{\mathrm{s}}$ representan los límites de humedad entre los que se encuentra la curva de retención. El contenido de agua a saturación $\left(\theta_{\mathrm{s}}\right)$ puede obtenerse experimentalmente y en teoría su valor coincide con el de la porosidad, pero en la práctica es inferior debido a la presencia de aire atrapado o disuelto. El contenido de agua residual $\left(\theta_{r}\right)$ también se puede obtener experimentalmente a partir de la determinación del contenido de agua de una muestra de suelo secada al aire.

Otro punto característico de la curva de retención es el potencial de entrada de aire, que corresponde al valor límite de succión para el cual los poros de mayor tamaño empiezan a perder agua. El parámetro a de la ecuación de van Genuchten está estrechamente ligado a la inversa del potencial de entrada de aire. Un valor alto de a (por ejemplo en suelos arenosos) implica que los suelos se desecan rápidamente ante pequeños valores de succión, mientras que un valor bajo (por ejemplo suelos arcillosos) indica una desecación lenta a medida que la succión se incrementa. En Carsel y Parrish (1988) pueden encontrarse tabulados los valores de los parámetros de la ecuación de van Genuchten en función de la textura, obtenidos a partir del promedio de un gran número de muestras.

El parámetro $\mathrm{n}$ también está correlacionado con la textura, aumentado a medida que aumenta el porcentaje de arena. La forma de la curva de retención es afectada por el valor de $\mathrm{n}$, reduciéndose la pendiente en su zona central al aumentar el valor de $\mathrm{n}$ (aumentar el contenido de arena) lo que se traduce en que a igual aumento de la succión el contenido de agua se reduce más en un suelo de textura gruesas que en un suelo de textura fina.

El parámetro $L$ está relacionado con la conectividad de los poros. Con el objetivo de simplificar la parametrización, se le atribuye el valor de 0,5 obtenido a partir del promedio de los valores determinados para muchos suelos (Mualem, 1976).

El modelo Hydrus 2D requiere de la introducción de los parámetros hidráulicos del suelo objeto de estudio. La obtención de dichos parámetros experimentalmente en laboratorio es costosa, por lo que generalmente se obtienen a partir de las llamadas funciones de edafotransferencia (FET).

Las funciones de edafotrasnferencia se denominan así porque transforman propiedades de los suelos fácilmente medibles en los parámetros hidráulicos del suelo (Schaap et al., 2001).

Se pueden distinguir dos tipos de FET, las que utilizan técnicas estadísticas de regresión y las que utilizan redes neuronales y tratamiento de bases de datos (Vereecken et al., 2008). En las del primer tipo, los parámetros hidráulicos se determinan mediante ecuaciones de regresión lineal y no lineal que relacionan los datos de textura, densidad aparente y/o contenido de materia orgánica (entre otras posibles variables) con valores de humedad del suelo cuando el potencial es conocido, es decir, ciertos puntos de la curva de retención.

Las redes neuronales no requieren una ecuación de cálculo previamente establecida sino que utilizan procedimientos de calibración iterativos para relacionar las características de los suelos con las propiedades hidráulicas de sus bases de datos.

EI modelo ROSETTA (Schaap et al., 2001) está basado en FET de redes neuronales y es un programa ampliamente utilizado en modelización de los parámetros hidráulicos del suelo. Este software utiliza distintas bases de datos dependiendo del grado de detalle y calidad de los datos de entrada sobre características de suelo. Numerosos autores han utilizado el modelo ROSETTA para la estimación inicial de los parámetros hidráulicos de suelo, como Schott et al. (2000), Skaggs et al. (2004), Jiang et al. (2010), Jiménez-Martínez (2010), Kanzari et al., (2012a) entre otros. 
Skaggs et al. (2004) midieron la distancia al emisor y profundidad de bulbo húmedo obteniendo los parámetros hidráulicos del suelo mediante "Rosetta completo", es decir, con todos los datos de entrada que requiere (densidad aparente, porcentajes de arena, limo y arcilla, y el contenido de agua a 33 y $1500 \mathrm{kPa}$ ) y comparándolo con los parámetros de suelo obtenidos introduciendo sólo la textura de suelo. Comprobaron que la simulación obtenida por el modelo Hydrus con los parámetros de suelo obtenidos con todos los datos de entrada de Rosetta eran iguales que la simulación obtenida a partir de los datos texturales del suelo.

El modelo Hydrus 2D/3D tiene incorporado el modelo ROSETTA (Lite Versión 1.1) para calcular los parámetros hidráulicos del suelo en función de la clase textural de éste.

Para calibrar el modelo se ha medido el bulbo húmedo en un ensayo llevado a cabo en laboratorio en una urna de metacrilato. La urna tiene unas dimensiones de $70 \mathrm{~cm}$ de largo, $50 \mathrm{~cm}$ de alto y $5 \mathrm{~cm}$ de ancho. Paralelamente, en el modelo Hydrus se ha modelizado una geometría rectangular idéntica a la urna, de $70 \mathrm{~cm}$ de largo por $50 \mathrm{~cm}$ de alto para simular el flujo vertical en dos dimensiones en el plano (XZ). El perfil está compuesto de una capa homogénea de suelo arenoso de la misma textura que el ensayado en la urna ( $94 \%$ arena, $4 \%$ limo, $2 \%$ arcilla).

Una vez calibrado el modelo se ha simulado el frente húmedo que genera un gotero de $5 \mathrm{I} . \mathrm{h}^{-1} \mathrm{~m}^{-1}$ en un lomo típico del cultivo de fresa. Se ha adoptado una geometría plana, y se ha simulado la mitad de un lomo. El lomo presenta una profundidad de $50 \mathrm{~cm}$, con una anchura en coronación de $25 \mathrm{~cm}$ y de $35 \mathrm{~cm}$ en la base. Las condiciones de contorno fijadas han sido flujo variable en una longitud determinada para simular el flujo del emisor, ausencia de flujo en el resto de la geometría, ya que está acolchado con plástico dentro de un túnel y por tanto no influye el agua de lluvia, y drenaje libre en el límite inferior (figura 1).

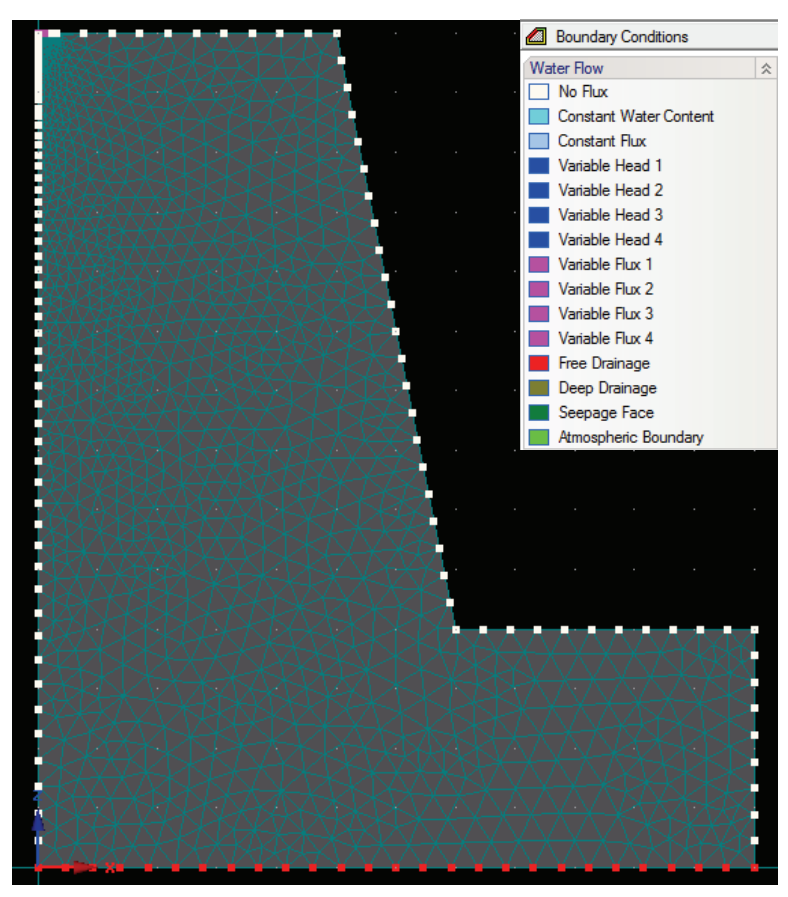

Figura 1. Geometría, malla y condiciones de contorno generadas para la simulación.

La textura del suelo corresponde al suelo de una finca comercial de fresa $(94 \%$ arena, $4 \%$ limos y $2 \%$ arcilla) obteniéndose los principales parámetros hidráulicos mediante el modelo ROSETTA.

Se ha fijado la profundidad y ancho radicular para cada periodo de cultivo simulado. En los meses de mayor demanda, de abril a junio, se ha fijado la profundidad radicular en 40 
$\mathrm{cm}$ y el radio máximo de raíces en $20 \mathrm{~cm}$. Estos datos están en la línea de los empleados por Gärdenäs et al. (2005) para simular el flujo de agua en el cultivo de fresa.

Debido a que el lomo se encuentra acolchado, se ha considerado que toda la evapotranspiración es debida a la transpiración de las plantas (Jiménez-Martínez, 2010). Igualmente no se considera que la lluvia influya en el contenido de humedad del suelo debido a que el cultivo se desarrolla en lomos acolchados bajo túnel.

El contenido inicial de agua se ha fijado para todo el perfil en $0,20 \mathrm{~cm}^{3} / \mathrm{cm}^{3}$, cercano a capacidad de campo para un suelo con alto contenido en arena, como puede ser un suelo arenoso, arenoso-franco, o franco-arenoso.

\section{Resultados y conclusiones.}

Se han simulado tres etapas o periodos del cultivo claramente diferenciados. En la fase inicial del cultivo (noviembre-enero) las necesidades hídricas del cultivo oscilan en torno a $1 \mathrm{~mm} /$ día. Debido a que los valores medios de $\mathrm{ET}_{\mathrm{c}}$ en esta etapa son muy bajos y unidos al hecho de que las plántulas en esta fecha tienen muy poco desarrollo vegetativo y radicular, no se ha considerado la $\mathrm{ET}_{\mathrm{c}}$ en la evolución de la humedad del suelo. El tiempo de riego necesario para satisfacer las necesidades del cultivo oscila entre los 10 y 20 minutos para una cinta de riego de $5 \mathrm{I} . \mathrm{h}^{-1} . \mathrm{m}$. De la simulación realizada con el modelo se desprende que el pulso efectivo de riego en esta primera fase de la campaña comienza con un tiempo de 10 minutos y se irá incrementando hasta un máximo de 20 minutos a medida que las plantas se van desarrollando y las raíces van creciendo. Este pulso se registra a $12 \mathrm{~cm}$ de profundidad y es inapreciable a $25 \mathrm{~cm}$, con lo que se consigue mantener la humedad en los primeros $12 \mathrm{~cm}$ de suelo, dónde se concentran las raíces en esta etapa.

En la etapa de mediados de campaña (febrero-marzo) el valor de $\mathrm{ET}_{\mathrm{c}}$ promedio dentro de invernadero asciende a $2,5 \mathrm{~mm} / \mathrm{di}$ í. El tiempo de riego requerido por el cultivo oscila entre los 20-40 minutos diarios para una cinta de riego de $5 \mathrm{I} . \mathrm{h}^{-1} . \mathrm{m}$. De la simulación realizada con el modelo se observa que el pulso efectivo de riego comienza con tiempos de riego cercanos a los 20-30 minutos que se pueden ir incrementando hasta 40 minutos a medida que aumentan las necesidades hídricas del cultivo y se va desarrollando el cultivo. Se observa que con estos pulsos de riego la humedad se registra a $12 \mathrm{~cm}$ y levemente a 25 $\mathrm{cm}$, pero no se registra a $40 \mathrm{~cm}$, favoreciendo la concentración de la humedad en los primeros $25 \mathrm{~cm}$ de suelo dónde se encuentran las raíces.

En la etapa de final de campaña (máxima demanda) los requerimientos hídricos del cultivo aumentan considerablemente desde principio de abril hasta mayo-junio en los que la demanda evaporativa se va incrementando de forma significativa. El valor medio de $E T_{c}$ estimado en estos meses asciende a $5 \mathrm{~mm} /$ día. Los tiempos de riego teóricos para satisfacer las necesidades del cultivo oscilan entre los 40-60 minutos diarios para una cinta de $5 \mathrm{I} . \mathrm{h}^{-1} . \mathrm{m}$. En esta etapa, los pulsos de riego típicos registrados en campo según la gestión habitual de los regantes aumentan considerablemente, siendo habitual aplicar pulsos de riego que oscilan entre 90 y 120 minutos, generalmente aplicados en dos pulsos de riego cercanos a una hora. Para aplicar la $\mathrm{ET}_{\mathrm{c}}$ del periodo punta se han simulado tres estrategias:

1. Un único pulso de 1 hora de duración (hora de inicio 10 de la mañana).

2. Dos pulsos de 30 minutos (con horas de inicio 10 de la mañana y 3 de la tarde).

3. Tres pulsos de 20 minutos diarios (con horas de inicio a las 10, 15 y 18 horas).

En esta época final, en la que las temperaturas comienzan a ascender de manera considerable y debido a que la planta se encuentra en plena fase de fructificación, se ha previsto que el riego sea diario. Se han simulado un periodo de una semana promedio del mes de mayo y un volumen de agua aplicado equivalente a la ETc del cultivo en esa época. En las tres simulaciones el volumen total de agua diaria aplicada ha sido de 1 litro diario y 7 litros semanales. Se ha considerado que las raíces en esta etapa tienen una profundidad 
máxima de $40 \mathrm{~cm}$ encontrándose la máxima densidad de raíces a $20 \mathrm{~cm}$, y que el radio máximo es de $20 \mathrm{~cm}$, con la máxima densidad de raíces a $10 \mathrm{~cm}$.

En la figura 2 se observa que para las simulaciones llevadas a cabo el bulbo húmedo que se genera se mantiene en los primeros $20 \mathrm{~cm}$ del suelo, aunque si bien en las tres estrategias después de una semana aumenta la humedad en todo el lomo. En la fase de redistribución de la humedad en el suelo se observa como unen los frentes de humedad de la parte superficial del lomo con la humedad existente en las capas más profundas. 
AERYD
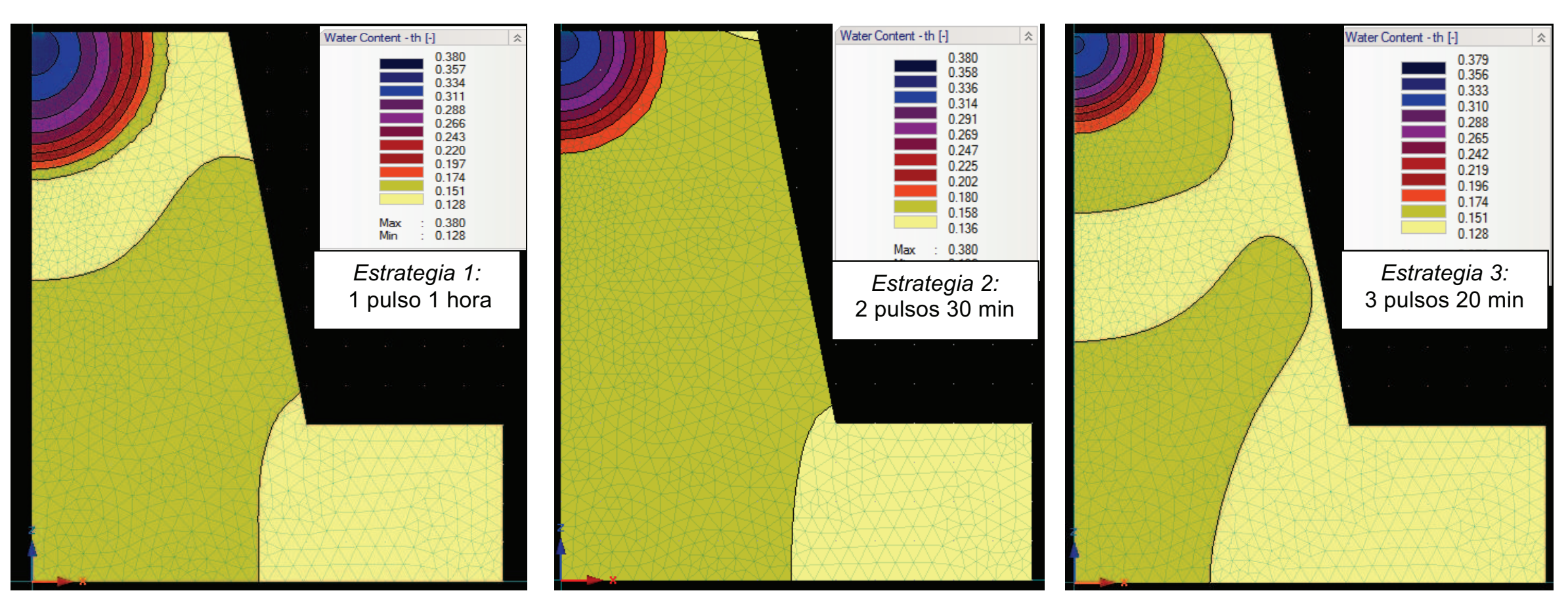

Figura 2. Simulación del contenido de agua en el suelo $\left(\mathrm{cm}^{3} / \mathrm{cm}^{3}\right)$ al final del último evento de riego para las estrategias 1, 2 y 3
XXXIII Congreso Nacional de Riegos

Universitat Politècnica de València, Valencia 2015

DOI:http://dx.doi.org/10.4995/CNRiegos.2015.1472 

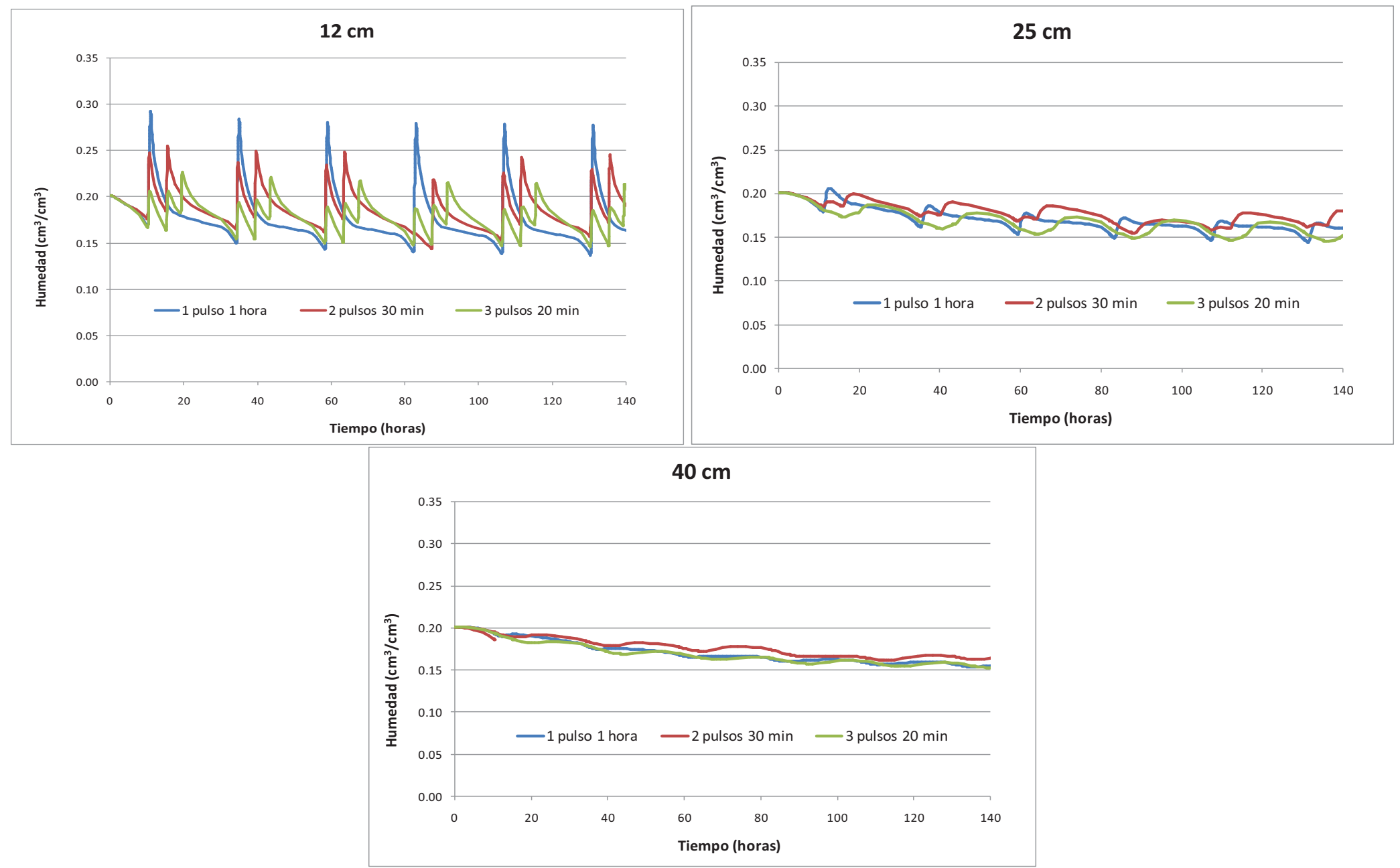

Figura 3. Contenido volumétrico de humedad en el suelo $\left(\mathrm{cm}^{3} / \mathrm{cm}^{3}\right)$ a 12, 25 y $40 \mathrm{~cm}$ para las estrategias 1,2 y 3 
En la figura 3 se puede apreciar que la humedad que se alcanza a los $12 \mathrm{~cm}$ es mayor para un pulso de 1 hora (estrategia 1) alcanzando valores de humedad en torno a $0,28 \mathrm{~cm}^{3} / \mathrm{cm}^{3}$. En la estrategia 2 se registran valores de humedad cercanos a $0,25 \mathrm{~cm}^{3} / \mathrm{cm}^{3}$ pero durante más tiempo que en la estrategia 1. La estrategia 3 mantiene la humedad en valores cercanos a $0,2 \mathrm{~cm}^{3} / \mathrm{cm}^{3}$ durante más tiempo a lo largo del día que las estrategias 1 y 2. En los tres casos la humedad mínima simulada es similar y alcanza el valor de 0,15 $\mathrm{cm}^{3} / \mathrm{cm}^{3}$.

La humedad del pulso de riego se registra a los $25 \mathrm{~cm}$ de la superficie del lomo para las tres estrategias de riego. En los tres casos la humedad se mantiene en el mismo intervalo de $0,15-0,20 \mathrm{~cm}^{3} / \mathrm{cm}^{3}$. A $40 \mathrm{~cm}$ de la superficie del lomo los cambios de humedad son muy leves para las tres estrategias, manteniéndose en los tres casos por encima de $0,15 \mathrm{~cm}^{3} / \mathrm{cm}^{3}$.

En resumen, el comportamiento de las tres estrategias de riego diseñadas para aplicar la $\mathrm{ET}_{\mathrm{c}}$ del cultivo en el período de máxima demanda, a $25 \mathrm{~cm}$ y $40 \mathrm{~cm}$ es similar. La mayor diferencia se encuentra en los primeros $12 \mathrm{~cm}$ del perfil. En esa franja la estrategia 3, que aplica 3 pulsos de 20 minutos, presenta la ventaja de que mantiene la humedad cercana a un valor medio de $0,2 \mathrm{~cm}^{3} / \mathrm{cm}^{3}$ durante más tiempo a lo largo del día, evitando picos acentuados de humedad. Aplicar las necesidades de cultivo en varios pulsos de riego de menor duración es una estrategia muy recomendable especialmente en suelos con poca capacidad de retención de la humedad, como son los suelos destinados al cultivo de fresa en la zona de estudio.

\section{Conclusiones.}

Se ha comprobado que el modelo Hydrus es una herramienta adecuada para simular el movimiento del agua en el suelo, y por tanto poder definir diferentes estrategias de riego eficientes que eviten las pérdidas de agua por percolación y de nutrientes por lixiviación. En el caso del cultivo de fresa se han simulado diferentes estrategias de riego para aplicar la $\mathrm{ET}_{\mathrm{c}}$ del cultivo con emisores de $5 \mathrm{I}^{-\mathrm{h}^{-1}} \mathrm{~m}$ en tres etapas diferentes del cultivo, inicio, mediados, y final de campaña. Por término medio se necesitan tiempos de riego que oscilan entre los 10-20 minutos al inicio de la campaña y 40-60 minutos al final de campaña, en pulsos de no más de 20 minutos.

Los tiempos de riego aplicados en campo por los agricultores locales son superiores, ya que se aplica por término medio entre 1,5 y 2 veces la $E T_{c}$. Se recomienda revisar la programación del riego, adaptando el riego a las necesidades del cultivo, manteniendo la humedad en los primeros $30-40 \mathrm{~cm}$ de suelo y evitando así la pérdida de agua por percolación así como la posible contaminación del acuífero por lixiviados.

\section{Bibliografía}

Arbat Pujolràs, G., 2005. Desarrollo y validación de un modelo de simulación de la dinámica del agua en el suelo. Aplicación al diseño agronómico y al manejo en riego localizado. Tesis Doctoral, Universidad de Lleida.

Carsel, R. and Parrish, R., 1998. Developing joint probability of soil water retention characteristics. Water Resources Research, 24 (5), 755-769.

Feddes, R. A., Kowalik, P.J., Zaradny, H., 1978. Simulation of Field Water Use and Crop Yield, John Wiley \& Sons, New York, NY. 
Gärdenäs, A.I., Hopmans, J.W., Hanson, B.R., Šimůnek, J., 2005. Two-dimensional modeling of nitrate leaching for various fertigation scenarios under micro-irrigation. Agricultural Water Management 7, 219.242.

Jiang, S., Pang, L., Buchan, G., Šimůnek, J., Noonan, M., Close, M. 2010. Modelling water flow and bacterial transport in undisturbed lysimeters under irrigations of dairy shed effluent and water using Hydrus-1D. Water Research 44, 1050-1061.

Jiménez-Martínez, J. 2010. Aquifer recharge from intensively irrigated farmland. Several approaches. PhD Thesis, Technical University of Catalonia.

Kanzari, S., Hachicha, M., Bouhlila, R., Battle-Sales, J., 2012a. Characterization and modeling of water movement and salts transfer in a semi-arid region of Tunisia (Bou Hajla, Kairouan)-Salinization risks of soils an aquifers. Computers and Electronics in Agriculture, $86,34-42$.

Mualem, Y., 1976. A new model for predicting the hydraulic conductivity of unsaturated porous media. Water Resour. Res. 12: 513-522

Samadanfiard, S., Sadraddini, A.A., Nazemi, A. H., Provenzano, G., Kisi, Ö., 2012. Estimating soil wetting patterns for drip irrigation using genetic programming. Spanish Journal of Agricultural Research 10 (4), 1155-1166.

Schaap, M.G., Leij, F.J., van Genuchten, T., 2001. ROSETTA: a computer program for estimating soil hydraulic parameters with hierarchical pedotransfer functions. Journal of Hydrology, 251, 163-176.

Scott, R., Shuttleworth, W., Keefer, T., Warrick, A. 2000. Modeling multiyear observations of soil moisture recharge in the semiarid American Sothwest. Water Res. Research, 36, $\mathrm{n}^{\circ} 8$, 2233-2247.

Šimůnek, J., Šejna, M., van Genuchten, M. Th., 1999. The Hydrus-2D software package for simulating two-dimensional movement of water, heat and multiple solutes in variably saturated media, version 2.0. Rep. IGCWMC-TPS-53, Int. Ground Water Model. Cen., Colo. Sch. Of Mines, Golden,CO, p.251.

Šimůnek, J., van Genuchten, M. Th., Šejna, M., 2006. The Hydrus software package for simulating two and three-dimensional movement of water, heat, and multiple solutes in variably-saturated media. Technical manual, Vers. 1.0. PC Progresss, Prague, Czech Republic.

Šimůnek, J., van Genuchten, M.Th., Šejna, M., 2008. Development and applications of the Hydrus and STANMOD software packages, and related codes. Vadose Zone, J. 7 (2), 587600.

Skaggs, T.H., Trout, T.J., Šimůnek, J., Shouse, P.J. 2004. Comparison of Hydrus-2D Simulations of Drip irrigation with Experimental Observations. Journal of Irrigation and Drainage Engineering 130:4 (304). 
Vereecken, H., Huisman, J., Bogena, H., Vanderborght, J., Vrugt, J., Hopmans, J. 2008. On the value of soil moisture measurements in vadose zone hydrology: A review. Water Resources Research, 44, W00D06.

van Genuchten, M. Th., 1980. A closed-form equation for predicting the hydraulic conductivity of unsaturated soils. Soil Sci. Soc. Am. J., 44, 892.898.

van Genuchten, M. Th., 1987. A numerical model for water and solute movement in and below the root zone. Res. Rep. 121. U.S. Salinity Lab., Riverside, C.A. 